\title{
Etude De L'exposition Des Banques Islamiques Au Taux D’intérêts Et De L’implication Sur La Gestion Actif-Passif
}

\author{
Mohamed Wail Aaminou \\ Ecole Mohammadia d’Ingénieurs, Université Mohammed V
}

Aziz Moutahaddib

Ecole Nationale de Commerce et de Gestion

Université Ibn Tofail

doi: 10.19044/esj.2017.v13n10p263 URL:http://dx.doi.org/10.19044/esj.2017.v13n10p263

\begin{abstract}
Theoretically, Islamic banks should not be exposed to the risk of interest rate fluctuations. Indeed, in the literature, Islamic finance is considered a finance without interest that stands out from conventional finance by means of profit sharing and losses.

In practice, although Islamic banking products are not directly based on interest, several studies mentioned in this article have shown that these banks are not completely immune to this kind of risk. Moreover, unlike conventional banks, Islamic banks do not have the same flexibility to cover this risk since they do not use interest.

So, the assessment of the level of exposure to interest risks and understanding its determinants is essential for studying the appropriate hedging strategies and the guarantee of stability of Islamic banks.
\end{abstract}

Keywords : Islamic banks, risks, interest rates, ALM

Résumé

Théoriquement, les banques islamiques ne devraient pas être exposées aux risques de fluctuation des taux d'intérêts. En effet, dans la littérature, la finance islamique est considérée comme une finance sans intérêts qui se démarque de la finance conventionnelle par des instruments de partage des profits et des pertes.

Dans la pratique, bien que les produits bancaires islamiques ne se basent pas directement sur les intérêts, plusieurs études évoquées, dans le cadre de cet article, ont démontré que ces banques ne sont pas complètement à l'abri de ce genre de risques. Par ailleurs, contrairement aux banques classiques, les 
banques islamiques n’ont pas la même souplesse pour couvrir ce risque puisqu'elles n'utilisent pas l'intérêt.

Ainsi, l'évaluation du niveau d'exposition aux risques d'intérêts et la compréhension de ses déterminants sont primordiaux dans l'étude des stratégies de couverture appropriées et la garantie de la stabilité des banques islamiques.

Mots clés : Banques islamique, risques, taux d’intérêt, ALM

\section{Introduction}

Les actifs financiers islamiques dépassent 1,650 milliards de dollars (Reuters, 2014) avec un taux de croissance annuel moyen de 15\% (Nazim and Bennie, 2012). Les services financiers islamiques sont proposés par plus de 251 banques, 281 compagnies d'assurance and 791 fonds d'investissements (Reuters, 2014). Les banques islamiques sont des entreprises à but lucratif qui, à l'instar des banques conventionnelles, se basent dans leurs modèles d'affaires sur l’intermédiation financière par la collecte des dépôts et la distribution des financements à la clientèle de détail et aux entreprises (Guéranger, 2009). Cependant, les banques islamiques opèrent suivant des principes distinctifs basés sur le droit commercial islamique dans la conduite de leurs activités. Les principaux principes concernent l'interdiction de la rémunération du capital par l'intérêt, l'interdiction de l'incertitude excessive (gharar) et le conditionnement du gain par la prise de risque (Ayub, 2009).

A la base des éléments présentés ci-dessus, les banques islamiques devraient être immunisées contre le risque de taux d’intérêts qui se définit comme « le risque encouru en cas de variation des taux d'intérêt du fait de l'ensemble des opérations de bilan et hors-bilan, à l'exception, le cas échéant, des opérations soumises aux risques de marché » (Banque_de_France, 1997). Cependant, plusieurs études empiriques réalisées notamment en Malaisie et en Indonésie documentent clairement l'exposition des banques islamiques au risque d'intérêts.

En effet, dans une première étude réalisée en Malaisie entre 1999 et 2006, Kassim et al. (2009) démontrent que les banques islamiques sont sensibles aux changements de la politique monétaire plus que les banques conventionnelles. Les auteurs se basent sur le modèle Vecteur Autoregressif pour démontrer que les changements des taux d'intérêts directeurs ont un impact sur les encours des dépôts et des financements des banques islamiques. Plus précisément, quand les taux d’intérêts sont élevés, les déposants des banques islamiques migrent vers les banques conventionnelles et vis versa. Ces résultats sont cohérents avec les conclusions d'autres études réalisées par Kader and Leong (2009) et Haron and Ahmad (2000) sur le 
marché bancaire Malaisien et par Kasri and Kassim (2009) sur le marché bancaire Indonésien. Dans une étude similaire, conduite en Malaisie entre 1997 et 2008 par Zainol and Kassim (2010) les auteurs mettent en évidence les liens de causalité et de corrélation entre le taux de rémunération des comptes à terme (trois mois) des banques conventionnelles et les taux de rendement sur les dépôts d'investissements ainsi que l'encours de ensemble des dépôts des banques islamiques. Ces mêmes liens de causalité sont également mis en évidence par Kaleem and Isa (2003).

Dans ce qui suit, nous présentons dans le deuxième paragraphe brièvement les raisons de cette exposition et son impact sur les banques islamiques. Ensuite nous proposerons, dans le troisième paragraphe, une méthodologie pour gérer le risque d’intérêts dans le cadre de la politique « Gestion Actif/Passif » des banques islamiques.

\section{Explication de l'exposition des banques islamiques aux taux d'intérêts}

A l'exception de l'Iran et du Soudan les banques islamiques opèrent dans des marchés financiers duaux où elles cohabitent avec les banques conventionnelles(Khoutem Ben JEDIDIA, 2013). De ce fait, la clientèle cible des deux banques reste commune, ce qui constitue une courroie de transmissions entre les sphères islamiques et conventionnelles (Zainol and Kassim, 2010). Concernant les critères de choix des banques islamiques, il est à noter qu'en plus de l'argument de conformité avec charia (Amin et al., 2013, Echchabi and Nafiu Olaniyi, 2012, Hin et al., 2011, Sayani and Miniaoui, 2013, Wajdi Dusuki and Irwani Abdullah, 2007), la rentabilité des dépôts et les coûts des financements pèsent considérablement dans la décision des clients (Abduh and Omar, 2012, Echchabi and Nafiu Olaniyi, 2012, Hasan et al., 2012, Marimuthu et al., 2010, Metawa and Almossawi, 1998, Sayani and Miniaoui, 2013).

Ainsi, la clientèle qui cherche à maximiser ses gains en cherchant les options d' " arbitrage » entre les deux offres bancaires expose les banques islamiques au risque commercial déplacé qui « résulte de la gestion des comptes d'investissement participatifs non restrictifs. Il découle plus spécifiquement du comportement de leurs titulaires, qui, insatisfaits de la rémunération aléatoire offerte par leur banque, peuvent retirer leurs fonds, faisant courir un grave risque d'illiquidité à l'établissement. Sous pression commerciale, les banques islamiques sont obligées de violer le principe de partage des profits avec les titulaires des comptes d'investissement et lissent ainsi les revenus associés à ces comptes pour atténuer le risque commercial déplacé » (Toumi and Laurent Viviani, 2013)

Les banques islamiques, (Aldo Lévy, 2012) alignent leurs pratiques sur celles de la finance classique, pour diminuer le risque de volatilité de ses clients. Par exemple, les taux de marge de Mourabaha sont sensiblement les 
mêmes que pour les crédits ... Elles recourent également à des ajustements des marges de Mourabaha et Ijara wa Ictina sur les taux d'intérêts pratiqués par les banques classiques.

Il nous semble, à cet effet, que l'utilisation des taux intérêts comme base de calcul des marges des financements (Ayub, 2009) contribue et renforce ce comportement des clients. Il est vrai que les normes de l'AAOIFI $\left({ }^{20}\right)$ autorisent le recours des banques islamiques à des benchmarks conventionnels tels que le $\operatorname{LIBOR}\left({ }^{21}\right)$ et l'EURIBOR $\left({ }^{22}\right)$ faute d'alternatives. Cependant, cette option crée de la confusion auprès des clients et encourage le comportement d’arbitrage précédemment cité.

\section{Méthodologie pour gérer les risques d'intérêt dans le cas des banques islamiques}

La gestion Actif-Passif ( J.W.Bitner et A.Robert Goddard, 1992) a pour objectif de gérer le risque de taux pesant sur le bilan de la banque, gérer les besoins de liquidité relative à l'activité bancaire, préserver le capital de la banque et augmenter le capital de la banque. Elle vise (M. Dubernet, 1997) à cantonner dans les limites consciemment déterminées les conséquences négatives éventuelles des risques financiers, principalement, risque de liquidité, risque de taux et risque de change.

L’objectif (Bessis, 1995) est d’assurer la pérennité de l’établissement en planifiant son développement et son financement et ne doit pas se fixer pour objectif de maximiser la rentabilité de l'établissement.

Selon Brom (2009), la gestion actif-passif est destinée à mesurer et à gérer les risques découlant de facteurs tels que la volatilité des taux de change et des taux d'intérêt et la disponibilité de fonds (liquidité). Ces facteurs échappent au contrôle de l'organisation et sont tous fonction du marché de l'offre et de la demande monétaires dans l'économie mondiale. Un processus de gestion actif-passif efficace permet aux organisations de minimiser les risques inhérents au bilan en équilibrant autant que possible, en fonction de la politique de gestion des risques, des flux financiers et des échéances des actifs et des dettes. Lorsque les échéances et les flux financiers des actifs et des dettes sont parfaitement alignés, le risque financier disparaît. En pratique, il est difficile d'assurer un équilibre parfait. Il est donc important de mesurer les asymétries et de fixer des plafonds, ou

\footnotetext{
${ }^{20}$ Accounting and Auditing Organization for Islamic Financial Institutions

${ }^{21}$ Le LIBOR est une série de taux de référence du marché monétaire de différentes devises. Son nom a été formé à partir des initiales de la dénomination anglaise London interbank offered rate (en français : « taux interbancaire pratiqué à Londres »).

${ }^{22}$ L'Euribor désigne un groupe de taux d'intérêt de la devise Euro largement utilisés en Europe. Ils sont, avec l'Eonia, les principaux taux de référence du marché monétaire de la zone euro.
} 
des limites, à l'étendue du risque que l’organisation est disposée à prendre. Ainsi, la compréhension et la gestion des risques des taux d'intérêt est une composante essentielle de la gestion actif-passif.

Typiquement, le bilan d'une banque islamique est composé de la manière suivante. Les ratios présentés sont ceux de la Banque Zitouna (Tunisie) en Décembre 2013.

\begin{tabular}{|c|c|}
\hline Actif & Passif \\
\hline Financements clientèle & Dépôts clientèle \\
Mourabaha (83\%) & Comptes courants non rémunérés (35\%) \\
Ijara (12\%) & Comptes épargne et dépôts \\
Autres financements : Istisnaa, & d'investissement (65\%) \\
moucharaka... (5\%) & Sukuk et autres financements \\
Immobilisations & Capitaux propres \\
Autres actifs & \\
\hline
\end{tabular}

Concernant les postes de l'actif, les grandes masses sont constituées premièrement par les financements mourabaha et ensuite par les financements Ijara. D’une manière générale, les flux monétaires générés par ces financements à un instant donné ne sont pas impactés par le changement des taux d'intérêt. Il est à noter que certains financements ijara peuvent être indexés sur un benchmark et donc peuvent être sensibles aux changements des taux d'intérêt. Concernant les postes du passif, les grandes masses sont constituées par les comptes de dépôts rémunérés et ensuite par les comptes courants non rémunérés.

En l'absence du risqué commercial déplacé présenté dans le deuxième paragraphe, la problématique actif-passif d'un point de vue risque d'intérêt ne se poserait pas. En fait, les déposants des comptes d'épargne et d'investissement sont rémunérés en fonction de la rentabilité des actifs. En réalité, une partie des déposants compare entre la rémunération des banques islamiques et celles servies par les banques conventionnelles et sont à l'affut d'opportunités d'arbitrage. Ainsi, si les taux d'intérêt augmentent, les clients des banques islamiques se retrouvent désavantagés, ce qui oblige la banque islamique à servir une rémunération supérieure, ce qui affecte sa liquidité et sa rentabilité. Inversement, la banque islamique profiterait d'un niveau supérieur de rentabilité des actifs pour constituer des réserves afin de faire face à des futurs mouvements défavorables des taux. Pour résumer et pour faire l'analogie avec le conventionnel, la banque islamique se retrouve globalement avec des emplois à taux fixes financés avec des ressources mixtes (fixes et variables).

Dans les banques conventionnelles, une stratégie de couverture contre ce genre d'exposition est de recourir à des instruments de couverture en hors bilan tels que les swaps de taux ou des options sur les taux d'intérêts. Une autre stratégie serait de convertir le mode de rémunération des dépôts 
vers un profile de rémunération fixe. Dans l'environnement financier islamique, très peu d'instruments de couverture en hors bilan existent et quand ils existent, ils sont souvent controversés d'un point de vue de la conformité charia. Par ailleurs, il n’est pas possible de rémunérer les dépôts par un taux fixe. (Ayub, 2009).

Cependant en droit commercial islamique, il existe pourtant deux grands types d'accords contractuels qui présentent des caractéristiques similaires aux dérivés et permettent la vente d’objets intangibles. Ces contrats ont pour nom SALAM (paiement anticipé et livraison différée) et ISTISNAA (financement progressif et livraison différée). Les principes qui les régissent sont les mêmes que ceux des contrats à terme conventionnels, mais ils demeurent, à certains égards, sujets à controverse aux yeux des juristes classiques (Guéranger- François, 2009).

Aussi et en réalité, les IFI recourent, parfois, à l'usage implicite et parfois même explicite des produits dérivés :

Implicitement : en restant à distance, certaines IFI sous-traitent ou délèguent cette gestion du risque de marché, auprès de banques conventionnelles soient à travers des MOURABAHA dans une optique de pure transfert de risque ou carrément des MOUCHARAKA, laissant le soin à son associé « conventionnel » d’opérer pour le compte de leur entreprise commune, les transactions nécessaires sur les dérivés pour assurer le «sale boulot» à sa place, sans qu’elles y soient directement impliquées.

Explicitement : Dans ce cadre, les juristes se penchent de plus en plus sur des produits ( tels que le KHIYAR, L'URBUN, LE WAÄD..) qui ont suscité beaucoup d’objections (Gharar et Maysir). Mais au regard du droit islamique, la licéité de ces produits peut être appréciée, d’une manière générale, en invoquant l'intérêt général (maslaha) de la communauté financière.

Face à ce risque, les banques islamiques aujourd'hui créent des réserves PER (Profit Equalization Reserves) et IRR (Investment Risk Reserve) pour lisser les profits et absorber les fluctuation du rendement des actifs comme indiqué par les normes de l'AAOIFI (Zainol and Kassim, 2010).

Bien qu’elle permette de gérer le risque à un certain degré, le recours à cette stratégie n'est pas toujours efficace. Par exemple, quand l'augmentation des taux continue pendant une longue période avec des actifs Mourabaha de longues maturités (financement l'immobilier). C'est ainsi que nous proposons trois pistes de solutions :

- Innovation financière: L'industrie financière islamique doit redoubler d'efforts au niveau de l'innovation portant sur des instruments de gestion des risques comme proposé par Kassim et al. (2009). L’effort d’innovation devrait être conduit en harmonie avec les objectifs et finalités 
chariatiques. La même recommandation concerne également la création de benchmarks de référence propres à l’industrie financière islamique

- $\quad$ Education financière des clients sur le fonctionnement des comptes d'investissements et du modèle de fonctionnement des banques islamiques. Plus précisément, les clients devraient comprendre que le profil d'un compte d'investissement islamique diffère d'un compte à terme conventionnel. Si cette compréhension est acquise, le recours aux réserves PER et IRR n’aura pas une aussi grande importance.

- $\quad$ Diversification des comptes de dépôt rémunérés. Il est opportun d'étudier la possibilité de définir plusieurs profils de comptes d'investissements différenciés par type de risques. Par exemple, une première catégorie de comptes d'investissement servira pour des financements avec un faible taux de risque et donc avec moins de rentabilité (financement de logements, véhicules...), une seconde pour des financements avec un niveau de risque et de rentabilité plus élevés (financement d'entreprises, projets...). Par ailleurs, la diversification des instruments de financement par les banques islamiques et le recours à des instruments de partage des profits et des pertes au lieu des instruments à marge fixe comme proposé par Zainol and Kassim (2010) facilitera la mise en place de cette recommandation.

\section{Conclusion}

Dans le présent travail, nous avons mis l'accent sur la problématique de l'exposition des banques islamiques aux risques de taux d'intérêt et sur les impacts de cette exposition sur la gestion Actif-Passif au sein de ces banques. Nous avons également proposé des recommandations pour réduire l'impact de ce risque. Ces recommandations portent sur l'innovation en ingénierie financière en gestion des risques, l'éducation du marché ainsi que le développement de nouveaux dépôts d'investissements.

Nous pensons que la thématique de l'exposition des banques islamiques aux risques de taux d'intérêt a besoin davantage d'études qualitatives et quantitatives pour bien appréhender ce type de risque et proposer les stratégie de couverture adéquates au delà du marché Malaisien et Asiatique.

\section{References:}

1. ABDUH, M. \& OMAR, M. A. 2012. Islamic-Bank Selection Criteria in Malaysia: An AHP Approach. Business Intelligence Journal, 5, 271-281.

2. AMIN, M., ISA, Z. \& FONTAINE, R. 2013. Islamic banks: Contrasting the drivers of customer satisfaction on image, trust, and 
loyalty of Muslim and non-Muslim customers in Malaysia. International Journal of Bank Marketing, 31, 79-97.

3. AYUB, M. 2009. Understanding Islamic Finance, John Wiley \& Sons.

4. BANQUE_DE_FRANCE 1997. Règlement no 97-02 du 21 février 1997 relatif au contrôle interne des établissements de crédit et des entreprises d'investissement. In: FRANCE, B. D. (ed.).

5. BROM, K. 2009. Gestion actif-passif pour les institutions de microfinance collectrices de dépôts. CGAP.

6. ECHCHABI, A. \& NAFIU OLANIYI, O. 2012. Malaysian consumers' preferences for Islamic banking attributes. International Journal of Social Economics, 39, 859-874.

7. GUÉRANGER, F. 2009. Finance islamique: une illustration de la finance éthique, Dunod.

8. HARON, S. \& AHMAD, N. 2000. The effects of conventional interest rates and rate of profit on funds deposited with Islamic banking system in Malaysia. International Journal of Islamic Financial Services, 1, 1-7.

9. HASAN, S. A., SUBHANI, M. I. \& OSMAN, M. 2012. Consumer criteria for the selection of an Islamic Bank: Evidence from Pakistan.

10. HIN, C. W., WEI, C. C., BOHARI, A. M. \& ABIDIN ADAM, M. Z. 2011. Bank selection criteria and service quality of Islamic banking: A comparison between Muslim and non-Muslim students and its effect on student's satisfaction.

11. KADER, R. A. \& LEONG, Y. K. 2009. The impact of interest rate changes on Islamic bank financing. International Review of Business Research Papers, 5, 189-201.

12. KALEEM, A. \& ISA, M. M. 2003. Casual relationship between Islamic and conventional banking instruments in Malaysia. International Journal of Islamic Financial Services, 4, 1-8.

13. KASRI, R. \& KASSIM, S. H. 2009. Empirical determinants of saving in the Islamic banks: evidence from Indonesia. Ahmad, N. and Haron, S.(2002)"Perceptions of Malaysian Corporate Customers Towards Islamic Banking Products and Services”, International Journal of Islamic Financial Services, 3, 13-29.

14. KASSIM, S. H., MAJID, M. S. A. \& YUSOF, R. M. 2009. Impact of monetary policy shocks on the conventional and Islamic banks in a dual banking system: Evidence from Malaysia. Journal of Economic Cooperation and Development, 30, 41-58.

15. Khoutem Ben JEDIDIA, l’intermédiation financière participative des banques islamiques, les cahiers de la Finance Islamique $\mathrm{N}^{\circ} 3$, Page 16,2013 
16. MARIMUTHU, M., WAI JING, C., PHEI GIE, L., PEY MUN, L. \& YEW PING, T. 2010. Islamic banking: selection criteria and implications. Global Journal of Human-Social Science Research, 10.

17. METAWA, S. A. \& ALMOSSAWI, M. 1998. Banking behavior of Islamic bank customers: perspectives and implications. International Journal of Bank Marketing, 16, 299-313.

18. NAZIM, A. \& BENNIE, G. 2012. World Islamic Banking Competitiveness Report 2012-2013. Ernst \& Young 2012,(December).

19. REUTERS, T. 2014. State of the global Islamic economy. Thomson Reuters 2014 Report.

20. SAYANI, H. \& MINIAOUI, H. 2013. Determinants of bank selection in the United Arab Emirates. International Journal of Bank Marketing, 31, 206-228.

21. TOUMI, K. \& LAURENT VIVIANI, J. 2013. Le risque lié aux comptes d'investissement participatifs: un risque propre aux banques islamiques. La Revue des Sciences de Gestion, 259, 131-142.

22. WAJDI DUSUKI, A. \& IRWANI ABDULLAH, N. 2007. Why do Malaysian customers patronise Islamic banks? International Journal of Bank Marketing, 25, 142-160.

23. ZAINOL, Z. \& KASSIM, S. H. 2010. An analysis of Islamic banks' exposure to rate of return risk. Journal of Economic Cooperation and Development, 31, 59-84. 